\title{
Renormalization in theories with modified dispersion relations: Weak gravitational fields
}

\author{
D. López Nacir, F.D. Mazzitelli* \\ Departamento de Física Juan José Giambiagi, Facultad de Ciencias Exactas y Naturales, UBA, Ciudad Universitaria, Pabellón I, 1428 Buenos Aires, Argentina
}

\section{A R T I C L E I N F O}

\section{Article history:}

Received 14 October 2008

Received in revised form 22 December 2008

Accepted 5 January 2009

Available online 19 January 2009

Editor: M. Cvetič

\section{PACS:}

04.60.Bc

$04.62 .+\mathrm{v}$

11.10.Gh

\begin{abstract}
A B S T R A C T
We consider a free quantum scalar field satisfying modified dispersion relations in curved spacetimes, within the framework of Einstein-Aether theory. Using a power counting analysis, we study the divergences in the adiabatic expansion of $\left\langle\phi^{2}\right\rangle$ and $\left\langle T_{\mu \nu}\right\rangle$, working in the weak field approximation. We show that for dispersion relations containing up to $2 s$ powers of the spatial momentum, the subtraction necessary to renormalize these two quantities on general backgrounds depends on $s$ in a qualitatively different way: while $\left\langle\phi^{2}\right\rangle$ becomes convergent for a sufficiently large value of $s$, the number of divergent terms in the adiabatic expansion of $\left\langle T_{\mu \nu}\right\rangle$ increases with $s$. This property was not apparent in previous results for spatially homogeneous backgrounds.
\end{abstract}

(C) 2009 Elsevier B.V. Open access under CC BY license.
There are theoretical and phenomenological motivations for studying quantum fields with modified dispersion relations (MDR) in the effective field theory framework for semiclassical gravity [18]. For example, in some inflationary models the expansion of the universe can last sufficiently long that length scales of cosmological interest today could have been sub-Planckian (or smaller than a critical length for which new physics could show up) at the beginning of inflation. In such models, the inflationary predictions could be affected by such unknown physics, giving rise to so-called trans-Planckian effects [5]. A similar argument in the context of black hole physics has motivated the analysis of the robustness of the Hawking effect [6] under the modification of the dispersion relation of the quantum fields. It has also been argued that transPlanckian effects could be relevant in the astrophysical context, as for example in the physics of ultra high energy cosmic rays [4].

In some models, the inclusion of fields satisfying MDR locally breaks Lorentz invariance. Nevertheless, one can include MDR while preserving a generally covariant metric theory for gravity by working in the framework of the Einstein-Aether theory [7]. In this theory, the general covariance is preserved by introducing a dynamical vector field $u^{\mu}$ called the aether field, which is constrained by means of a Lagrange multiplier to take a non-zero timelike value, $u^{\mu} u_{\mu}=-1$.

In the semiclassical approximation, a study of the consequences of changing the dispersion relation should involve a proper treatment of the divergences that appear in the expectation values of

\footnotetext{
* Corresponding author.

E-mail addresses: dnacir@df.uba.ar (D. López Nacir), fmazzi@df.uba.ar (F.D. Mazzitelli).
}

observables associated to the quantum fields. For example, a careful evaluation of the expectation value of the stress tensor is necessary to evaluate the backreaction of the quantum fields on the background metric, since this expectation value is the source term in the semiclassical equations for the metric.

The renormalization procedure for quantum fields satisfying the standard dispersion relations in curved backgrounds is well established [9-11]. Indeed, there are well known covariant methods of renormalization that can be implemented in principle in any spacetime metric. When applied to the expectation value of the square of the field $\left\langle\phi^{2}\right\rangle$, or to the mean value of the stress tensor $\left\langle T_{\mu \nu}\right\rangle$, one can obtain the associated renormalized quantities by making the subtractions:

$$
\begin{aligned}
& \left\langle\phi^{2}\right\rangle_{\text {ren }}=\left\langle\phi^{2}\right\rangle-\left\langle\phi^{2}\right\rangle^{(0)}-\cdots-\left\langle\phi^{2}\right\rangle^{\left(2 i_{\max }\right)}, \\
& \left\langle T_{\mu \nu}\right\rangle_{\text {ren }}=\left\langle T_{\mu \nu}\right\rangle-\left\langle T_{\mu \nu}\right\rangle^{(0)}-\cdots-\left\langle T_{\mu \nu}\right\rangle^{\left(2 j_{\max }\right)},
\end{aligned}
$$

where a superscript $2 l$ denotes the terms of adiabatic order $2 l$ of the corresponding expectation value (i.e., the terms containing $2 l$ derivatives of the metric). For the usual dispersion relation, in $n$ dimensions the subtraction involves up to the adiabatic orders $2 i_{\max }^{u}=2 \operatorname{int}(n / 2-1)$ for $\left\langle\phi^{2}\right\rangle$ and $2 j_{\max }^{u}=2 \operatorname{int}(n / 2)$ for the stress tensor, where $\operatorname{int}(x)$ is the integer part of $x$ (the superscript $u$ stands for usual).

In the case of MDR, the renormalization procedure have been investigated in some particular cases [12-14]. For scalar fields propagating in an $n$-dimensional spatially flat Robertson-Walker (RW) spacetime, in Ref. [12] we have considered the extension of the adiabatic subtraction scheme based on a WKB expansion of the field modes [15] to the case of generalized dispersion relation. There, the WKB expansion of the stress tensor was computed up 
to the fourth adiabatic order. With the use of dimensional regularization, it was shown that these adiabatic orders can be absorbed into a redefinition of the gravitational bare constants of the theory (i.e., the cosmological constant, the Newton constant and the coupling constants associated to terms quadratic in the curvature in the gravitational action), which correspond to the counterterms also required for the usual dispersion relation. However, this simple result is a peculiarity due to the symmetries of the spatially flat RW metric. Indeed, in Ref. [13] we have followed the same approach for the case of four-dimensional Bianchi type I metrics. Restricting the calculation up to the second adiabatic order, we have shown that new counterterms do appear, which involve the aether field in addition to the metric. For instance, a term proportional to $\left(\nabla_{\mu} u^{\mu}\right)^{2}$ in the aether Lagrangian is needed in order to absorb the divergences in $\left\langle T_{\mu \nu}\right\rangle^{(2)}$ (in addition to the usual Einstein-Hilbert action). The point is that in a spatially flat RW background these new counterterms are indistinguishable from the usual ones. Concretely, once evaluated in this background, the stress tensor obtained from the variation of the most general action for the aether field containing two derivatives, turns out to be proportional to the Einstein tensor. As there are strong constraints on the parameters associated to terms containing two derivatives of the vector field [8], the new counterterms of second adiabatic order should be carefully chosen to make the theory consistent with observation [13].

In both spatially flat Robertson-Walker and Bianchi type I backgrounds, given a MDR such that the frequency behaves as $\omega \sim|\vec{k}|^{s}$ for large values of $|\vec{k}|$, one can show that divergences appear up to $[12,13]$

$2 i_{\max }^{h}=2 \operatorname{int}\left(\frac{n-1}{2 s}-\frac{1}{2}\right), \quad 2 j_{\max }^{h}=2 \operatorname{int}\left(\frac{1}{2}+\frac{n-1}{2 s}\right)$,

where the superscript $h$ stands for (spatially) homogeneous. Note that the correct values for the usual dispersion relation are recovered for $s=1$. Eq. (2) suggests that the higher the power $s$ of $|\vec{k}|$ the milder would be the divergences. For example, in four dimensions, this result indicates that if $s \geqslant 4$ the divergences of the stress tensor are contained only in the zeroth adiabatic order. However, as will be shown below, this is valid only for the spatially homogeneous backgrounds considered so far.

In this Letter we will consider weak (but otherwise general) background fields, and use a power counting analysis to investigate up to which adiabatic order the subtractions in Eq. (1) have to be carried out in order to get finite, physically meaningful expectation values. In the case of interacting quantum fields in Minkowski spacetime, higher spatial derivatives improve the UV behavior of Feynman diagrams (see for instance [16]). Here, we will show that while such improvement also occurs for $\left\langle\phi^{2}\right\rangle$, this is not the case for $\left\langle T_{\mu \nu}\right\rangle$.

By adopting the semiclassical approximation we consider a massive quantum scalar field $\phi$ propagating in a classical curved spacetime and coupled to a classical aether. The classical action for the scalar field is given by $[17,18]$ :

$S_{\phi}=\int d^{n} x \sqrt{-g}\left(\mathcal{L}_{\phi}+\mathcal{L}_{\text {cor }}\right)$,

where $n$ is the spacetime dimension, $g=\operatorname{det}\left(g_{\mu \nu}\right), \mathcal{L}_{\phi}$ is the standard Lagrangian of a free scalar field

$\mathcal{L}_{\phi}=-\frac{1}{2}\left[g^{\mu \nu} \partial_{\mu} \phi \partial_{\nu} \phi+\left(m^{2}+\xi R\right) \phi^{2}\right]$,

with $R$ the Ricci scalar, $\mathcal{L}_{\text {cor }}$ is the corrective Lagrangian that gives rise to a generalized dispersion relation

$\mathcal{L}_{\text {cor }}=-\sum_{q, p \leqslant q} b_{q p}\left(\mathcal{D}^{2 q} \phi\right)\left(\mathcal{D}^{2 p} \phi\right)$, with $\mathcal{D}^{2} \phi \equiv \perp_{\mu}^{\lambda} \nabla_{\lambda}\left(\perp_{\gamma}^{\mu} \nabla^{\gamma} \phi\right)$, where $\perp_{\mu \nu} \equiv g_{\mu \nu}+u_{\mu} u_{v}$ and $\nabla_{\mu}$ is the derivative operator associated with $g_{\mu \nu}$.

After varying the action (3) with respect to $\phi$, one can write the equation for the associated Green's function $G\left(x, x^{\prime}\right)$ as

$$
\begin{aligned}
& {\left[\square-\left(m^{2}+\xi R\right)-\sum_{q, p \leqslant q} b_{q p}\left(H^{q} \mathcal{D}^{2 p}+H^{p} \mathcal{D}^{2 q}\right)\right] G\left(x, x^{\prime}\right)} \\
& \quad=-\frac{1}{\sqrt{|g|}} \delta\left(x-x^{\prime}\right),
\end{aligned}
$$

where $H$ is an operator given by

$H=\mathcal{D}^{2}+\nabla_{\alpha} a^{\alpha}+2 a^{\alpha} \nabla_{\alpha}$,

with $a^{\alpha} \equiv u^{\mu} \nabla_{\mu} u^{\alpha}$.

In the weak field approximation, we consider the linearized Eq. (6) about the flat background solution with Minkowski metric $\eta_{\mu \nu}$ and constant aether $\underline{u}_{\mu}$ [19]. Adopting Minkowski coordinates $\left(x^{0}, \ldots, x^{n-1}\right)$ such that $\underline{u}_{\mu}=\delta_{\mu}^{0},{ }^{1}$ the fields can be expanded as

$g_{\mu \nu}=\eta_{\mu \nu}+h_{\mu \nu}, \quad g^{\mu \nu}=\eta^{\mu \nu}-h^{\mu \nu}$,

$u_{\mu}=\delta_{\mu}^{0}+v_{\mu}, \quad u^{\mu}=-\delta_{0}^{\mu}-h^{0 \mu}+v^{\mu}$.

Here and in what follows, we keep only linear terms in $h_{\mu \nu}$ and $v_{\mu}$. The constraint $u_{\mu} u^{\mu}=-1$ yields $v^{0}=h^{00} / 2$. After choosing the Lorentz gauge

$\partial^{\mu} \bar{h}_{\mu \nu}=0$,

$\bar{h}_{\mu \nu}=h_{\mu \nu}-\frac{1}{2} \eta^{\mu \nu} h$,

with $h=h_{\rho}^{\rho}$, we obtain

$\sqrt{|g|} \square=\left(\eta^{\mu \nu}-\bar{h}^{\mu \nu}\right) \partial_{\mu} \partial_{\nu}$

$\sqrt{|g|} \sum_{q, p \leqslant q} b_{q p}\left(H^{q} \mathcal{D}^{2 p}+H^{p} \mathcal{D}^{2 q}\right)$

$=2 \sum_{q, p \leqslant q} b_{q p} \Delta^{q+p}+h \sum_{q, p \leqslant q} b_{q p} \Delta^{q+p}+\mathcal{K}$,

where $\Delta$ is the Laplacian and $\mathcal{K}$ is an operator of first order in the perturbation fields $h_{\mu \nu}$ and $v_{\mu}$. The explicit expression for the operator $\mathcal{K}$ can be easily found in the particular case in which the perturbation fields depend only on the time coordinate $x^{0}$. In the general case, although more complex, it can also be worked out. However, the expression in Eq. (10b) will be enough for our present purposes.

In order to solve Eq. (6), we first split $G=G^{0}+G^{1}$, where the superscripts refer to the order in $h_{\mu \nu}$ and $v_{\mu}$. The zeroth order propagator $G^{0}$ satisfies

$\left[-\partial_{0}^{2}+\Delta-m^{2}-2 \sum_{q, p \leqslant q} b_{q p} \Delta^{q+p}\right] G^{0}\left(x, x^{\prime}\right)=-\delta\left(x-x^{\prime}\right)$.

Hence, the corresponding Feynman propagator is

$G_{F}^{0}\left(x, x^{\prime}\right)=\int \frac{d^{n} k}{(2 \pi)^{n}} \frac{e^{i k\left(x-x^{\prime}\right)}}{\left[-k_{0}^{2}+\omega^{2}\left(|\vec{k}|^{2}\right)-i \epsilon\right]}$

where $k x=\eta_{\mu \nu} k^{\mu} x^{\nu}$, and

$\omega^{2}\left(|\vec{k}|^{2}\right)=m^{2}+|\vec{k}|^{2}+2 \sum_{q, p \leqslant q} b_{q p}(-1)^{q+p}|\vec{k}|^{2(q+p)}$,

with $\vec{k}=\left(k_{1}, \ldots, k_{n-1}\right)$.

1 Alternatively one can choose $\underline{u}^{\mu}=\delta_{0}^{\mu}$. However physical results will not depend on this (conventional) choice. 
Then, the first order correction to the propagator satisfies

$$
\begin{aligned}
& {\left[-\partial_{0}^{2}+\Delta-m^{2}-2 \sum_{q, p \leqslant q} b_{q p} \Delta^{q+p}\right] G^{1}\left(x, x^{\prime}\right)} \\
& =\left[\bar{h}^{\mu \nu} \partial_{\nu} \partial_{\mu}-\frac{\xi}{2} \eta^{\mu \nu}\left(\partial_{\nu} \partial_{\mu} h\right)+\frac{h}{2}\left(\omega^{2}(-\Delta)+\Delta\right)+\mathcal{K}\right] G^{0}\left(x, x^{\prime}\right),
\end{aligned}
$$

and the corresponding solution is

$$
\begin{aligned}
G_{F}^{1}\left(x, x^{\prime}\right)= & -\int d^{n} y G_{F}^{0}(x, y)\left[\bar{h}^{\mu \nu}(y) \partial_{\nu} \partial_{\mu}-\frac{\xi}{2} \eta^{\mu \nu}\left(\partial_{\nu} \partial_{\mu} h(y)\right)\right. \\
& \left.+\frac{h(y)}{2}\left(\omega^{2}(-\Delta)+\Delta\right)+\mathcal{K}(y)\right] G_{F}^{0}\left(y, x^{\prime}\right) .
\end{aligned}
$$

In what follows, for the sake of convenience, we work in Euclidean spacetime with $k_{n}=-i k^{0}$ and $x_{n}=-i x^{0}$. The Euclidean Green's function $G_{E}$ is given by $G_{F}=i G_{E}$.

For our power counting analysis we consider, as an example, only one contribution to $G_{E}^{1}$, which we choose to be

$g_{E}^{1}\left(x, x^{\prime}\right)=\frac{1}{2} \int d^{n} y h(y) G_{E}^{0}(x, y) \omega^{2}(-\Delta) G_{E}^{0}\left(y, x^{\prime}\right)$.

One can readily check that this term, along with the one proportional to $\bar{h}^{00}(y) \partial_{0} \partial_{0}$ and similar terms within $\mathcal{K}(y)$, are the most divergent ones. By replacing the zeroth order solution $G_{E}^{0}$ into this equation, introducing the Fourier transform of $h$,

$h(p)=\int d^{n} y e^{-i p y} h(y)$,

and performing some trivial integrations, we find

$$
\begin{aligned}
g_{E}^{1}\left(x, x^{\prime}\right)= & \frac{1}{2} \int \frac{d^{n} p}{(2 \pi)^{n}} e^{i p x} h(p) \int \frac{d^{n} k}{(2 \pi)^{n}} \\
& \times \frac{e^{i k\left(x-x^{\prime}\right)} \omega^{2}\left(|\vec{k}|^{2}\right)}{\left[\left(k_{n}+p_{n}\right)^{2}+\omega^{2}\left(|\vec{k}+\vec{p}|^{2}\right)\right]\left[k_{n}^{2}+\omega^{2}\left(|\vec{k}|^{2}\right)\right]} .
\end{aligned}
$$

In order to derive the superficial degree of divergence of each adiabatic order we start by expanding the integrand in Eq. (18) in powers of $p_{i}(i=1, \ldots, n)$,

$$
\begin{aligned}
g_{E}^{1}\left(x, x^{\prime}\right)= & \frac{1}{2} \int \frac{d^{n} p}{(2 \pi)^{n}} e^{i p x} h(p) \int \frac{d^{n} k}{(2 \pi)^{n}} \frac{e^{i k\left(x-x^{\prime}\right)} \omega^{2}\left(|\vec{k}|^{2}\right)}{\left[k_{n}^{2}+\omega^{2}\left(|\vec{k}|^{2}\right)\right]^{2}} \\
& \times\left(\frac{1}{1+\epsilon_{p}}\right) \\
= & \frac{1}{2} \int \frac{d^{n} p}{(2 \pi)^{n}} e^{i p x} h(p) \int \frac{d^{n} k}{(2 \pi)^{n}} \frac{e^{i k\left(x-x^{\prime}\right)} \omega^{2}\left(|\vec{k}|^{2}\right)}{\left[k_{n}^{2}+\omega^{2}\left(|\vec{k}|^{2}\right)\right]^{2}} \\
& \times \sum_{r=0}^{+\infty}\left(-\epsilon_{p}\right)^{r},
\end{aligned}
$$

where

$\epsilon_{p}=\frac{2 k_{n} p_{n}+p_{n}^{2}+\omega^{2}\left(|\vec{k}+\vec{p}|^{2}\right)-\omega^{2}\left(|\vec{k}|^{2}\right)}{k_{n}^{2}+\omega^{2}\left(|\vec{k}|^{2}\right)}$.

The expectation value $\left\langle\phi^{2}\right\rangle$ is given by the coincidence limit of $\operatorname{Re} G_{E}\left(=\operatorname{Im} G_{F}\right)$. In such limit, $g_{E}^{1}$ can be decomposed as

$$
\begin{aligned}
& g_{E}^{1}(x, x)=\frac{1}{2} \int \frac{d^{n} p}{(2 \pi)^{n}} e^{i p x} h(p) \mathcal{I}(p), \\
& \mathcal{I}(p)=\int \frac{d^{n-1} k}{(2 \pi)^{n-1}} \omega^{2}\left(|\vec{k}|^{2}\right) I(p,|\vec{k}|) \\
& I(p,|\vec{k}|)=\int \frac{d k_{n}}{2 \pi} \sum_{r=0}^{+\infty} \frac{\left(-\epsilon_{p}\right)^{r}}{\left[k_{n}^{2}+\omega^{2}\left(|\vec{k}|^{2}\right)\right]^{2}} .
\end{aligned}
$$

Notice that, due to the remnant symmetries, the terms with odd powers of $p_{n}$ or $|\vec{p}|$ do not contribute, so there will be no odd adiabatic order contributions.

From Eq. (21) it is possible to derive the superficial degree of divergence of $\mathcal{I}(p)$ for each term with a given power of $p_{i}$. However, it is more instructive to compute explicitly the integral in $k_{n}$. Moreover, in order to appreciate better the difference between the cases in which the background fields depend or not on $\vec{x}$, we analyze them separately.

For background fields that depend only on $x_{n}$, we replace in Eq. (21) the $n$-dimensional Fourier transform $h(p)$ by $h(p)=$ $(2 \pi)^{n-1} \delta^{n-1}(\vec{p}) h\left(p_{n}\right)$. Performing the integral in $k_{n}$ of Eq. (21c) it is easy to see that

$I(p,|\vec{k}|)=\sum_{i=0}^{+\infty} \frac{\alpha_{i}}{\omega^{3}\left(|\vec{k}|^{2}\right)}\left(\frac{p_{n}}{\omega\left(|\vec{k}|^{2}\right)}\right)^{2 i}$,

where $\alpha_{i}$ is independent on $\vec{k}$. Therefore, substituting Eq. (22) into Eq. (21b), by means of power counting, we find that when the MDR is such that $\omega\left(|\vec{k}|^{2}\right) \sim|\vec{k}|^{s}$ for large values of $|\vec{k}|$, convergence of the $2 i$-adiabatic order of $\mathcal{I}(p)$ is guaranteed if $-s(2 i+3)+2 s+$ $n-2<-1$. That is, given the highest power $s$ of $|\vec{k}|$ in the MDR and the spacetime dimension $n$, we expect that the maximum adiabatic order of $\left\langle\phi^{2}\right\rangle$ that contains divergences will be given by

$2 i_{\max }^{h}=2 \operatorname{int}\left(\frac{n-1}{2 s}-\frac{1}{2}\right)$.

This result coincides with the one given in Eq. (2). It is worth to remark that the same result can be obtained using the weighted power counting analysis described in Ref. [16].

For background fields that depend only on $\vec{x}$, we introduce the spatial Fourier transform $h(\vec{p})$ related to $h(p)$ by $h(p)=$ $(2 \pi) \delta\left(p_{n}\right) h(\vec{p})$. In this case, performing the integration in $k_{n}$ we obtain

$I(p,|\vec{k}|)=\sum_{r=0}^{+\infty} \frac{\beta_{r}}{\omega^{3}\left(|\vec{k}|^{2}\right)}\left(\frac{\omega^{2}\left(|\vec{k}+\vec{p}|^{2}\right)}{\omega^{2}\left(|\vec{k}|^{2}\right)}-1\right)^{r}$,

where $\beta_{r}$ does not depend on $\vec{k}$. This equation should be compared with Eq. (22). In this case, the odd powers in $|\vec{p}|$ vanish once the angular integrations in Eq. (21b) are carried out. For large values of $\vec{k}, \omega^{2}\left(|\vec{k}+\vec{p}|^{2}\right)-\omega^{2}\left(|\vec{k}|^{2}\right) \sim|\vec{k}|^{2 s-2}(\vec{p} \cdot \vec{k})$, then power counting indicates that the $2 i$-adiabatic order will be finite if $2 i>n-1-s$. Hence, in this case, we expect that divergences will appear up to the $2 i_{\max }^{g}$-adiabatic order of $\left\langle\phi^{2}\right\rangle$, where

$2 i_{\max }^{g}=2 \operatorname{int}\left(\frac{n-1-s}{2}\right)$.

Here the superscript $g$ stands for general, since it can be easily shown that this is also the superficial degree of divergence for arbitrary backgrounds (i.e. $h_{\mu \nu}=h_{\mu \nu}\left(\vec{x}, x_{n}\right), v_{\mu}=v_{\mu}\left(\vec{x}, x_{n}\right)$ ). Note that while the values of $2 i_{\max }^{g}$ given in this equation coincide with those obtained from Eq. (23) for $n \leqslant 4$, it is generally larger for higher number of dimensions. For example, for $n=5$ and $s=2$ we have $2 i_{\max }^{g}=2$ while $2 i_{\max }^{h}=0$.

We have performed our power counting analysis for the particular contribution to $G_{E}^{1}$ given in Eq. (16), and one may wonder if other contributions to the propagator could cancel the divergences in $g_{E}^{1}$. In order to check explicitly that this is not the case, let us consider a MDR of the form $\omega^{2}\left(|\vec{k}|^{2}\right)=m^{2}+|\vec{k}|^{2}+2 b_{11}|\vec{k}|^{4}$ and compute the operator $\mathcal{K}$ defined in Eq. (10). For background fields that depend only on $\vec{x}$, after performing some integrations in Eq. (15), we arrive at 


$$
\begin{aligned}
\left\langle\phi^{2}\right\rangle= & \operatorname{Re} G_{E}^{1}(x, x) \\
= & -\frac{1}{2} \int \frac{d^{n-1} p}{(2 \pi)^{n-1}} e^{i \vec{p} \cdot \vec{x}} \int \frac{d^{n-1} k}{(2 \pi)^{n-1}}\left\{\frac{\bar{h}_{00}}{\omega\left(|\vec{k}|^{2}\right)+\omega\left(|\vec{k}+\vec{p}|^{2}\right)}\right. \\
& \left.+\frac{h(\vec{p}) m^{2}+\xi p^{2} h(\vec{p})-2 \sum_{i, j=1}^{n-1} \bar{h}^{i j} k_{i} k_{j}+f_{k}(\vec{p})}{2 \omega\left(|\vec{k}|^{2}\right) \omega\left(|\vec{k}+\vec{p}|^{2}\right)\left[\omega\left(|\vec{k}|^{2}\right)+\omega\left(|\vec{k}+\vec{p}|^{2}\right)\right]}\right\},
\end{aligned}
$$

where, up to second order of $p_{i}$,

$$
\begin{aligned}
f_{k}(\vec{p})= & 2 b_{11}\left\{h(\vec{p})|\vec{k}|^{4}-h_{00}(\vec{p})\left[|\vec{p}|^{2}|\vec{k}|^{2}-2(\vec{p} \cdot \vec{k})^{2}\right]\right. \\
& \left.-2 \sum_{i, j=1}^{n-1} h^{i j} k_{i} k_{j}\left[|\vec{p}|^{2}+2|\vec{k}|^{2}+2 \vec{p} \cdot \vec{k}\right]\right\} .
\end{aligned}
$$

Then, we expand the terms between brackets in Eq. (26) up to second order in $p_{i}$ to obtain an integral expression for $\left\langle\phi^{2}\right\rangle^{(2)}$. By using dimensional regularization, we perform integrations by parts and discard surface terms to express all the integrals appearing in $\left\langle\phi^{2}\right\rangle^{(2)}$ in terms of only two of them. In this way, we obtain:

$$
\begin{aligned}
\left\langle\phi^{2}\right\rangle^{(2)}= & -\frac{\Omega_{n-1}}{8(2 \pi)^{n-1}}\left\{I_{3}\left(\xi-\frac{1}{6}\right) R^{1}\right. \\
& \left.+\tilde{I}_{3}\left[\frac{R^{1}}{6}+R_{00}^{1} \frac{3 n-7}{6(n-1)}\right]\right\},
\end{aligned}
$$

where the factor $\Omega_{n-1}=2 \pi^{(n-1) / 2} / \Gamma[(n-1) / 2]$ comes from the angular integration, $R^{1}=-\Delta h / 2, R_{00}^{1}=-\Delta h_{00} / 2$, and

$$
\begin{aligned}
& I_{3}=\int_{0}^{\infty} d x \frac{x^{\frac{(n-3)}{2}}}{\omega^{3}(x)}, \\
& \tilde{I}_{3}=\int_{0}^{\infty} d x \frac{x^{\frac{(n-1)}{2}}}{\omega^{3}(x)} \frac{d^{2} \omega^{2}(x)}{d x^{2}} .
\end{aligned}
$$

Here we see that while the integral $I_{3}$ converges in $n=5$ dimensions, there appears a new integral $\tilde{I}_{3}$ which is proportional to $b_{11}$ and diverges as $n \rightarrow 5$. Moreover, the divergence proportional to $R_{00}^{1}$ is not purely geometric. Indeed, it can be covariantly written in terms of the metric and the aether field since, to linear order in the perturbation fields, $R_{00}^{1}=R_{\mu \nu} u^{\mu} u^{v}=\nabla_{\mu} a^{\mu}$. On the other hand, when $\left\langle\phi^{2}\right\rangle^{(2)}$ is computed for background fields that depend only on the time coordinate, the second term in Eq. (28) does not appear and, in agreement with Eq. (23), this adiabatic order is finite. However, as cancellations are not to be expected for general background fields, divergences will generally appear up to the $2 i_{\text {max }}^{g}$-adiabatic order given in Eq. (25).

The differences between the two cases we are considering are better evidenced when the power counting analysis is applied to the stress tensor of the scalar field. The expectation value of the Euclidean stress tensor of the scalar field can be expressed as the coincidence limit of a nonlocal derivative operator applied to the two-point function $G_{E}^{1}\left(x, x^{\prime}\right)$. For example, the coincidence limit of the derivative with respect to $x_{n}$ and $x_{n}^{\prime}$ of $G_{E}^{1}\left(x, x^{\prime}\right)$ will contribute to the stress tensor. For our analysis let us consider the following contribution:

$$
\begin{aligned}
& {\left[\partial_{n} \partial^{\prime}{ }_{n} g_{E}^{1}\left(x, x^{\prime}\right)\right]_{x=x^{\prime}}=\frac{1}{2} \int \frac{d^{n} p}{(2 \pi)^{n}} e^{i p x} h(p) \mathcal{T}(p),} \\
& \mathcal{T}(p)=\int \frac{d^{n-1} k}{(2 \pi)^{n-1}} \omega^{2}\left(|\vec{k}|^{2}\right) T(p,|\vec{k}|), \\
& T(p,|\vec{k}|)=\int \frac{d k_{n}}{2 \pi} \sum_{r=0}^{+\infty} \frac{k_{n}^{2}\left(-\epsilon_{p}\right)^{r}}{\left[k_{n}^{2}+\omega^{2}\left(|\vec{k}|^{2}\right)\right]^{2}} .
\end{aligned}
$$

When the background fields depend only on $x_{n}$ we find that $T(p,|\vec{k}|)$ can be written as

$T(p,|\vec{k}|)=\sum_{j=0}^{+\infty} \frac{\gamma_{j}}{\omega\left(|\vec{k}|^{2}\right)}\left(\frac{p_{n}}{\omega\left(|\vec{k}|^{2}\right)}\right)^{2 j}$,

with $\gamma_{j} k$-independent. After substituting this expression into Eq. (30b) we obtain that divergences are contained in terms with up to $2 j_{\max }^{h}$ powers of $p_{n}$, where

$2 j_{\max }^{h}=2 \operatorname{int}\left(\frac{1}{2}+\frac{n-1}{2 s}\right)$,

in agreement with Eq. (2).

On the other hand, for background fields that depend only on $\vec{x}$ we obtain

$T(p,|\vec{k}|)=\sum_{r=0}^{+\infty} \frac{\zeta_{r}}{\omega\left(|\vec{k}|^{2}\right)}\left(\frac{\omega^{2}\left(|\vec{k}+\vec{p}|^{2}\right)}{\omega^{2}\left(|\vec{k}|^{2}\right)}-1\right)^{r}$,

where $\zeta_{r}$ does not depend on $k$. Recalling that odd powers in $|\vec{p}|$ do not contribute and using again that $\omega^{2}\left(|\vec{k}+\vec{p}|^{2}\right)-\omega^{2}\left(|\vec{k}|^{2}\right) \sim$ $|\vec{k}|^{2 s-2}(\vec{p} \cdot \vec{k})$ for large values of $\vec{k}$, we arrive at

$2 j_{\max }^{g}=2 \operatorname{int}\left(\frac{n-1+s}{2}\right)$,

whence we see that, contrary to the previous case, $2 j_{\max }^{g}$ increases with $s$. For instance, for $n=4$ and $s=1,2$ divergences appear up to the fourth adiabatic order, while for $s=3$ the sixth adiabatic order is also divergent. Hence, we expect that for $s \geqslant 3$ the renormalization of the expectation value of the stress tensor will require counterterms of adiabatic order higher than four.

Analogously to the case of $\left\langle\phi^{2}\right\rangle$, for a general background we do not expect that divergence cancellations occur. Therefore, we conclude that the subtraction in Eq. (1) should be performed up to the adiabatic orders $2 i_{\max }^{g}$ and $2 j_{\max }^{g}$ given in Eqs. (25) and (34), respectively. In particular, in order to renormalize the semiclassical Einstein-Aether equations it will be necessary to introduce all possible counterterms constructed with $g_{\mu \nu}$ and $u_{\mu}$ up to the $2 j_{\max }^{g}$-adiabatic order.

\section{Acknowledgements}

This work has been supported by Universidad de Buenos Aires, CONICET and ANPCyT.

\section{References}

[1] A. Kostelecký, S. Samuel, Phys. Rev. D 39 (1989) 683; A. Kostelecký, Phys. Rev. D 69 (2004) 105009.

[2] R. Gambini, J. Pullin, Phys. Rev. D 59 (1999) 124021; J. Alfaro, H.A. Morales-Tecotl, L.F. Urrutia, Phys. Rev. Lett. 84 (2000) 2318.

[3] D.A.R. Dalvit, F.D. Mazzitelli, C. Molina-Paris, Phys. Rev. D 63 (2001) 084023.

[4] G. Amelino-Camelia, arXiv: 0806.0339;

T. Jacobson, S. Liberati, D. Mattingly, Phys. Rev. D 67 (2003) 124011; R. Lehnert, Phys. Rev. D 68 (2003) 085003.

[5] R.H. Brandenberger, hep-ph/9910410; R.H. Brandenberger, J. Martin, Mod. Phys. Lett. A 16 (2001) 999; J. Martin, R.H. Brandenberger, Phys. Rev. D 63 (2001) 123501; J.C. Niemeyer, Phys. Rev. D 63 (2001) 123502.

[6] W. Unruh, Phys. Rev. Lett. 46 (1981) 1351; W. Unruh, Phys. Rev. D 51 (1995) 2827;

S. Corley, T. Jacobson, Phys. Rev. D 54 (1996) 1568 W. Unruh, R. Schützhold, Phys. Rev. D 71 (2005) 024028; W. Unruh, R. Schützhold, arXiv: 0804.1686 [gr-qc].

[7] T. Jacobson, D. Mattingly, Phys. Rev. D 63 (2001) 041502(R); T. Jacobson, D. Mattingly, Phys. Rev. D 64 (2001) 024028.

[8] B.Z. Foster, T. Jacobson, Phys. Rev. D 73 (2006) 064015; T. Jacobson, arXiv: 0801.1547 [gr-qc].

[9] R.M. Wald, Quantum Field Theory in Curved Spacetime and Black Hole Thermodynamics, University of Chicago Press, Chicago, 1994. 
[10] N.D. Birrell, P.C.W. Davies, Quantum Fields in Curved Space, Cambridge Univ. Press, Cambridge, 1982

[11] S.M. Fulling, Aspects of Quantum Field Theory in Curved Spacetime, Cambridge Univ. Press, Cambridge, 1989.

[12] D. López Nacir, F.D. Mazzitelli, C. Simeone, Phys. Rev. D 72 (2005) 124013; D. López Nacir, F.D. Mazzitelli, Phys. Rev. D 76 (2007) 024013.

[13] D. López Nacir, F.D. Mazzitelli, Phys. Rev. D 78 (2008) 044001.

[14] M. Rinaldi, Phys. Rev. D 76 (2007) 104027;

M. Rinaldi, Phys. Rev. D 78 (2008) 024025.

[15] L. Parker, S.A. Fulling, Phys. Rev. D 9 (1974) 341;
L. Parker, S.A. Fulling, Ann. Phys. (NY) 87 (1974) 176;

S.A. Fulling, L. Parker, B.L. Hu, Phys. Rev. D 10 (1974) 3905;

N.D. Birrell, Proc. R. Soc. London 361 (1978) 513;

P.R. Anderson, L. Parker, Phys. Rev. D 36 (1987) 2963;

T.S. Bunch, J. Phys. A 13 (1980) 1297.

[16] D. Anselmi, M. Halat, Phys. Rev. D 76 (2007) 125011

[17] M. Lemoine, M. Lubo, J. Martin, J.P. Uzan, Phys. Rev. D 65 (2001) 023510

[18] Throughout we set $c=1$ and use the sign convention denoted $(+++)$ in C.W. Misner, K.S. Thorne, J.A. Wheeler, Gravitation, Freeman, San Francisco, 1973.

[19] T. Jacobson, D. Mattingly, Phys. Rev. D 70 (2004) 024003. 